\title{
COMPUESTOS BIOACTIVOS Y ACTIVIDAD ANTIOXIDANTE DE SEMILLAS DE QUINUA PERUANA (Chenopodium quinoa W.)
}

\author{
Zanhy Valencia ${ }^{* a}$, Fernando Cámara $^{\mathrm{a}}$, Karina Ccapa $^{\mathrm{b}}$, Policarpo Catacora ${ }^{\mathrm{b}}$, Fredy Quispe ${ }^{\mathrm{b}}$
}

\begin{abstract}
RESUMEN
Se investigaron azúcares reductores, compuestos bioactivos y actividad antioxidante de 24 accesiones de quinua de la colección nacional del INIA Perú. Las semillas mostraron fenólicos totales entre 0,783 a 3,437 mg GAE/g, flavonoides totales entre 0,199 y 1,029 $\mathrm{mg} \mathrm{CE} / \mathrm{g}$ muestra, betacianinas y betaxantinas en cantidades no significativas, y azúcares reductores entre 30,973 y 88,278 equivalentes $\mathrm{mg}$ de glucosa/g muestra. La actividad antioxidante según DPPH y ABTS mostraron diferencias significativas entre las diferentes semillas de quinua estudiadas.
\end{abstract}

Palabras clave: Compuestos bioactivos, capacidad antioxidante, betalaínas, flavonoides.

\section{BIOACTIVE COMPOUNDS AND ANTIOXIDANT ACTIVITY FROM PERUVIAN QUINOA SEEDS (Chenopodium quinoa W.)}

\begin{abstract}
We investigated reducing sugars, bioactive compounds and antioxidant activity of 24 accessions of quinoa of the national collection of INIA Peru. The seeds showed total phenolic between 0,783 to $3,437 \mathrm{mg}$ GAE/g, total flavonoids between 0,199 and $1,029 \mathrm{mg}$ $\mathrm{CE} / \mathrm{g}$ sample, betacyanins and betaxanthins in amounts not significant and reducing sugars between 30,973 and $88,278 \mathrm{mg}$ of glucose equivalent/g sample. The antioxidant activity by DPPH and ABTS showed significant differences between different quinoa seeds studied.
\end{abstract}

Key words: Bioactive compounds, antioxidant activity, betalains, flavonoids.

\section{INTRODUCCIÓN}

La región de los Andes, cuna de las grandes civilizaciones como la Tiahuanacota y la Incaica es considerada centro de origen de muchas especies nativas como la quinua (Chenopodium

\footnotetext{
${ }^{* a}$ Departamento de Bromatología y Tecnología de los Alimentos Universidad de Córdoba, Carretera N-IV, Km 396, 14014 Córdoba, España email: zanhyvr@hotmail.com

${ }^{\mathrm{b}}$ Instituto Nacional de Innovación Agraria - INIA, Av. La Molina 1981 Lima, Perú
} 
quinoa W.), se cultivó durante miles de años y fue el principal alimento de las culturas antiguas de los Andes, por lo que está distribuido en diferentes zonas agroecológicas de la región, en la actualidad la quinua se encuentra en franco proceso de expansión porque representa un gran potencial para mejorar las condiciones de vida de la población de los Andes y del mundo moderno ${ }^{1}$. La composición nutricional de la quinua y sus compuestos bioactivos como los fenólicos y betalaínas pueden diferir según los ecotipos (grupos de cultivares definidos de acuerdo a su distribución, ecológica, agronómica y morfológica) ${ }^{2}$. Los polifenoles, incluyendo los ácidos fenólicos, flavonoides y taninos, son compuestos bioactivos que hacen que los metabolitos secundarios de la planta contribuyan a diversas actividades fisiológicas antimicrobianas, antinflamatorias, antitumorales y efectos anticancerígenos. Este beneficio fitoquímico, muy significativo, difiere en los diferentes cultivares de la misma planta ${ }^{3}$. Aparte de los macronutrientes, los estudios en quinua están enfocados en sus compuestos bioactivos de naturaleza hidrofílica como los ácidos fenólicos, flavonoides, actividad antioxidante y su potencial beneficio para la salud ${ }^{3}$. Por tal motivo el presente estudio tiene por objetivos determinar las diferencias entre compuestos bioactivos y actividad antioxidante de las semillas estudiadas e identificar accesiones con buenas características, para que así sean explotadas para el mejoramiento de la quinua.

\section{PARTE EXPERIMENTAL}

\section{Materiales y reactivos}

Semillas correspondientes a 24 accesiones de la Colección Nacional de Quinua del Instituto Nacional de Innovación Agraria Perú (B381, A114, A121, A122, A123, A124, A134, A142, A163, A174, A182, A221, A222, A231, A232, A243, A262, A263, A264, A282, A291, A131, A322, A331) se evaluaron en la investigación. Todos los reactivos y solventes fueron de grado analítico de las marcas Merck y Sigma Chemical Co.

\section{Métodos}

\section{Humedad}

Muestras molidas $(\varnothing<0,5 \mathrm{~mm})$ de las diferentes accesiones de quinua se evaluaron en el equipo Mettler Toledo (USA) ${ }^{4}$.

\section{Azúcares reductores}

Los azúcares reductores se determinaron según el método del ácido dinitrosalisílico (DNS) según Najmus ${ }^{5}$, con algunas modificaciones: $0,2 \mathrm{~mL}$ del extracto acuoso de las muestras se mezclaron con $0,8 \mathrm{~mL}$ del reactivo DNS y la solución resultante se calentó en baño María a 100 ${ }^{\circ} \mathrm{C}$ por $15 \mathrm{~min}$, después del tiempo señalado la solución se enfrió y se agregaron $5 \mathrm{~mL}$ de agua destilada para su evaluación, finalmente se midió la absorbancia en el espectrofotómetro UVVis (SPEKOL 1500, Alemania) a una longitud de onda a $540 \mathrm{~nm}$, y los azúcares reductores se obtuvieron a partir de una curva de calibración de glucosa entre los 100 y $1000 \mathrm{mg} / \mathrm{L}$ $\left(r^{2}=0,9933, y=0,0002 x-0,0288\right)$. 


\section{Preparación de los extractos}

Los extractos de EtOH-H $\mathrm{H}_{2} \mathrm{O}$ (1:1) para el análisis de compuestos fenólicos totales, actividad AA, flavonoides y betalaínas se prepararon de la siguiente manera: alrededor de $100 \mathrm{mg}$ de muestra molida $(\varnothing<0,5 \mathrm{~mm})$ se extrajeron con $10 \mathrm{~mL}$ de solución hidroalcohólica durante 12 horas a $4{ }^{\circ} \mathrm{C}$ por maceración, posteriormente los extractos se filtraron a través de un filtro de membrana de $0,45 \mu \mathrm{m}$ para su análisis. Adicionalmente, $100 \mathrm{mg}$ de muestra molida se extrajeron con $5 \mathrm{~mL}$ de solución $\mathrm{MeOH}: \mathrm{H}_{2} \mathrm{O}(2: 1)$ durante 60 minutos a $50{ }^{\circ} \mathrm{C}$, los extractos se filtraron a través de un filtro de membrana de $0,45 \mu \mathrm{m}$ y se enrazaron a un volumen de $10 \mathrm{~mL}$ con la misma solución, para su análisis. Para azúcares reductores, se utilizaron las mismas condiciones de extracción utilizando agua como solvente y una centrifugación adicional a $13000 \mathrm{rpm}$ durante $30 \mathrm{~min}$ a $5{ }^{\circ} \mathrm{C}$, el sobrenadante se almacenó a $4{ }^{\circ} \mathrm{C}$ hasta su evaluación.

\section{Fenólicos totales}

Los compuestos fenólicos totales se determinaron según Dini ${ }^{6}$ y Quispe ${ }^{7}$ : 0,1 mL del extracto se mezcló con 7,9 mL $\mathrm{H}_{2} \mathrm{O}$ destilada y $0,5 \mathrm{~mL}$ del reactivo de Folin Ciocalteu, se agitó y después se agregó $1,5 \mathrm{~mL}$ de $\mathrm{Na}_{2} \mathrm{CO}_{3}$ al $20 \%$, la solución se dejó en reposo 2 horas y al finalizar el tiempo se midió la absorbancia a $765 \mathrm{~nm}$ en el espectrofotómetro UV-Vis (SPEKOL 1500, Alemania). Los fenólicos totales se expresaron como equivalentes mg de ácido gálico GAE/g, a partir de una curva de calibración $\left(\mathrm{r}^{2}=0,9999, \mathrm{y}=0,0011 \mathrm{x}\right)$.

\section{Capacidad antioxidante}

La capacidad antioxidante de las diferentes muestras se evaluaron según 1,1-difenilo-2picril-hidrazilo (DPPH) y 2,2'-azino-bis-(3-etilbenzotiazolina-6-ácido sulfónico) (ABTS). Para DPPH en resumen: $0,3 \mathrm{~mL}$ de extracto hidroalcohólico (1:1) se mezcló con $2,7 \mathrm{~mL}$ de solución EtOH de DPPH, se agitó y dejó en reposo por $30 \mathrm{~min}$, al término de los cuales se midió la absorbancia a $517 \mathrm{~nm}$ en el espectrofotómetro UV-Vis (SPEKOL 1500, Alemania), la capacidad antioxidante se reportó como equivalentes mg de ácido gálico (GAE)/g muestra, a partir de una curva de calibración del \% de inhibición vs ácido gálico, $\left(\mathrm{r}^{2}=0,999, \mathrm{y}=4,6937 \mathrm{x}\right)^{8}$; en el caso de expresar la capacidad antioxidante como equivalentes Trolox, los extractos EtOH- $\mathrm{H}_{2} \mathrm{O}$ (1:1) y $\mathrm{MeOH}-\mathrm{H}_{2} \mathrm{O}$ (2:1), se hizo reaccionar con la solución EtOH de Trolox preparado entre 5 y $47 \mathrm{mg} / \mathrm{L}$, los que se graficaron con la diferencia de absorbancia obtenida para el DPPH, $\left(r^{2}=0,9997, y=0,009 x\right.$ para los extractos EtOH- $H_{2} O ; r^{2}=0,9981, y=0,0111 x$ para los extractos $\left.\mathrm{MeOH}-\mathrm{H}_{2} \mathrm{O}(2: 1)\right)$, y los resultados de actividad antioxidante se expresó como $\mu \mathrm{mol}$ Trolox/100 g muestra. En el caso del ABTS, el reactivo se disolvió en agua a una concentración de $7 \mathrm{mM}$ y el radical se produce por la adición de persulfato de potasio 2,45 mM, después de tiempos de reposo bajo oscuridad entre 12 a 16 horas, la solución se diluyó hasta una absorbancia de $0,7(+0,02)$ a $734 \mathrm{~nm}$ antes de su uso; para la evaluación de la actividad antioxidante $300 \mu \mathrm{L}$ de cada extracto se mezclaron con $3 \mathrm{~mL}$ de solución de ABTS, la mezcla se dejó en reposo por 7 min en la oscuridad, al término de los cuales se midió la absorbancia a $734 \mathrm{~nm}$ en el espectrofotómetro UV-Vis (SPEKOL 1500, Alemania) y la capacidad antioxidante se reportó como equivalentes $\mu$ mol Trolox $/ 100 \mathrm{~g}$ muestra (TEAC) a partir de curvas de calibración para los extractos EtOH- $\mathrm{H}_{2} \mathrm{O}(1: 1)\left(\mathrm{r}^{2}=0,9993, \mathrm{y}=0,012 \mathrm{x}\right) \mathrm{y}$ $\mathrm{MeOH}-\mathrm{H}_{2} \mathrm{O}(2: 1),\left(\mathrm{r}^{2}=0,9991, \mathrm{y}=0,0113 \mathrm{x}\right)^{9}$. 


\section{Flavonoides totales}

Los flavonoides totales se midieron según Dini ${ }^{6}$, para lo cual $0,5 \mathrm{~mL}$ del extracto $\mathrm{EtOH}-\mathrm{H}_{2} \mathrm{O}$ (1:1) de la muestra se mezclaron con $2 \mathrm{~mL} \mathrm{H}_{2} \mathrm{O}$ destilada y $0,15 \mathrm{~mL}$ de $\mathrm{NaNO}_{2}(5 \mathrm{~g} / 100 \mathrm{~mL})$, después de $5 \mathrm{~min}$ se agregó $0,15 \mathrm{~mL}$ de solución acuosa de $\mathrm{AlCl}_{3}(10 \mathrm{~g} / 100 \mathrm{~mL})$, y luego de 6 minutos se mezcló con $1 \mathrm{~mL}$ de $\mathrm{NaOH}(1 \mathrm{~N})$, finalmente la solución se enrasó con 1,2 mL de $\mathrm{H} 2 \mathrm{O}$ destilada a $5 \mathrm{~mL}$, se agitó la solución. La concentración de flavonoides totales se determinó a partir de la absorbancia de la solución a $510 \mathrm{~nm}$ en el espectrofotómetro UV-Vis (SPEKOL 1500, Alemania) se expresó como mg equivalente de catequina/g de muestra, a partir de una curva de calibración utilizando catequina como estándar entre 20 y $100 \mathrm{mg} / \mathrm{L}$ $(\mathrm{r} 2=0,9995, \mathrm{y}=0,0032 \mathrm{x})$.

\section{Betalaínas}

Las betalaínas se determinaron según von $\mathrm{Elbe}^{10}$, con algunas modificaciones: $0,2 \mathrm{~g}$ de la muestra se extrajo con $10 \mathrm{~mL}$ de buffer fosfato a $\mathrm{pH} 6,5$, la mezcla se agitó 2 horas a temperatura ambiente y oscuridad, al finalizar el tiempo señalado, las muestras se centrifugaron a $13000 \mathrm{rpm}$ a la temperatura de $5{ }^{\circ} \mathrm{C}$ por 30 minutos, finalmente se midieron las absorbancias de las muestras a 538, 476 y $600 \mathrm{~nm}$ en el espectrofotómetro UV-Vis (SPEKOL 1500, Alemania) y la determinación de betacianinas y betaxantinas resulta de reemplazar los valores de absorbancia en las expresiones: $\mathrm{x}=1,095(\mathrm{a}-\mathrm{c}), \mathrm{y}=\mathrm{b}-\mathrm{z}-\mathrm{x} / 3,1 \mathrm{y} \mathrm{z}=$ a-x; donde: $\mathrm{a}=$ absorbancia a $538 \mathrm{~nm}, \mathrm{~b}=$ absorbancia a $476 \mathrm{~nm}, \mathrm{c}=$ absorbancia a $600 \mathrm{~nm}, \mathrm{x}=$ absorbancia debido a betacianina, $\mathrm{y}=$ absorbancia debido a betaxantinas, $\mathrm{y} z=$ absorbancia debido a impurezas.

\section{Análisis estadístico}

Los datos se reportaron como media \pm desviación estándar. El software estadístico Infostat Versión 2015 se utilizó para el análisis estadístico de los datos experimentales. El análisis de varianza de un factor de variabilidad y prueba de significancia de Tukey $(\mathrm{p} \leq 0,05)$ se utilizó dentro del análisis estadístico ${ }^{11}$.

\section{RESULTADOS Y DISCUSIÓN}

Los resultados de las 24 accesiones de quinua, se presentan en la tabla 1. 
Tabla 1. Humedad, azúcares reductores, compuestos fenólicos, flavonoides, betacianinas y betaxantinas de las muestras de quinua.

\begin{tabular}{|c|c|c|c|c|c|c|}
\hline $\begin{array}{c}\text { Código } \\
\text { Accesión }\end{array}$ & $\begin{array}{l}\text { Humedad } \\
(\%) \\
* * \\
\end{array}$ & $\begin{array}{l}\text { Azúcares } \\
\text { reductores } \\
(\mathrm{mg} / \mathrm{g})^{* * * *}\end{array}$ & $\begin{array}{c}\text { Compuestos } \\
\text { fenólicos } \\
\text { (GAE mg/g) } \\
*_{*} *\end{array}$ & $\begin{array}{c}\text { Flavonoides } \\
\text { totales } \\
(\mathrm{mg} / \mathrm{g})^{* * *}\end{array}$ & $\begin{array}{c}\text { Betacianinas } \\
(\mathrm{mg} / 100 \mathrm{~g}) \\
* *\end{array}$ & $\begin{array}{c}\text { Betaxantinas } \\
(\mathrm{mg} / 100 \mathrm{~g}) \\
* *\end{array}$ \\
\hline B381 & $\begin{array}{l}8,81 \pm \\
0,13\end{array}$ & $\begin{array}{l}30,973 \pm \\
0,570\end{array}$ & $\begin{array}{l}2,739 \pm \\
0,052\end{array}$ & $\begin{array}{l}0,336 \pm \\
0,188\end{array}$ & $\begin{array}{l}0,278 \pm \\
0,025\end{array}$ & $\begin{array}{c}1,139 \pm \\
0,060\end{array}$ \\
\hline A114 & $\begin{array}{l}8,75 \pm \\
0,06\end{array}$ & $\begin{array}{l}75,848 \pm \\
2,402\end{array}$ & $\begin{array}{l}2,873 \pm \\
0,138\end{array}$ & $\begin{array}{l}0,321 \pm \\
0,229\end{array}$ & $\begin{array}{l}0,628 \pm \\
0,003\end{array}$ & $\begin{array}{l}7,552 \pm \\
0,133\end{array}$ \\
\hline A 121 & $\begin{array}{l}8,54 \pm \\
0,18 \\
\end{array}$ & $\begin{array}{l}47,393 \pm \\
0,143\end{array}$ & $\begin{array}{l}2,537 \pm \\
0,145 \\
\end{array}$ & $\begin{array}{l}0,645 \pm \\
0,076\end{array}$ & $\begin{array}{l}0,449 \pm \\
0,029\end{array}$ & $\begin{array}{l}5,009 \pm \\
0,015\end{array}$ \\
\hline A 122 & $\begin{array}{l}8,40 \pm \\
0,11 \\
\end{array}$ & $\begin{array}{l}38,205 \pm \\
1,211 \\
\end{array}$ & $\begin{array}{l}2,229 \pm \\
0,000\end{array}$ & $\begin{array}{l}0,664 \pm \\
0,075 \\
\end{array}$ & $\begin{array}{l}0,470 \pm \\
0,021\end{array}$ & $\begin{array}{l}4,983 \pm \\
0,064 \\
\end{array}$ \\
\hline A123 & $\begin{array}{l}8,27 \pm \\
0,17 \\
\end{array}$ & $\begin{array}{l}69,198 \pm \\
0,885\end{array}$ & $\begin{array}{l}0,783 \pm \\
0,052\end{array}$ & $\begin{array}{l}0,554 \pm \\
0,152\end{array}$ & $\begin{array}{l}0,500 \pm \\
0,028\end{array}$ & $\begin{array}{l}4,677 \pm \\
0,097\end{array}$ \\
\hline A 124 & $\begin{array}{l}8,52 \pm \\
0,13\end{array}$ & $\begin{array}{l}66,614 \pm \\
0,742\end{array}$ & $\begin{array}{l}3,327 \pm \\
0,181\end{array}$ & $\begin{array}{l}1,029 \pm \\
0,172\end{array}$ & $\begin{array}{l}0,883 \pm \\
0,028\end{array}$ & $\begin{array}{l}13,760 \pm \\
0,339 \\
\end{array}$ \\
\hline A 134 & $\begin{array}{l}9,05 \pm \\
0,02 \\
\end{array}$ & $\begin{array}{l}58,895 \pm \\
1,779 \\
\end{array}$ & $\begin{array}{l}2,537 \pm \\
0,114 \\
\end{array}$ & $\begin{array}{l}0,931 \pm \\
0,164 \\
\end{array}$ & $\begin{array}{l}0,738 \pm \\
0,029 \\
\end{array}$ & $\begin{array}{l}8,426 \pm \\
0,114 \\
\end{array}$ \\
\hline A 142 & $\begin{array}{l}8,91 \pm \\
0,19\end{array}$ & $\begin{array}{l}61,996 \pm \\
3,162\end{array}$ & $\begin{array}{l}2,712 \pm \\
0,091\end{array}$ & $\begin{array}{l}0,922 \pm \\
0,154\end{array}$ & $\begin{array}{l}0,584 \pm \\
0,023\end{array}$ & $\begin{array}{l}6,105 \pm \\
0,039\end{array}$ \\
\hline A163 & $\begin{array}{l}8,89 \pm \\
0,21 \\
\end{array}$ & $\begin{array}{l}64,614 \pm \\
0,943\end{array}$ & $\begin{array}{l}1,414 \pm \\
1,245\end{array}$ & $\begin{array}{l}0,393 \pm \\
0,103\end{array}$ & $\begin{array}{l}0,359 \pm \\
0,028\end{array}$ & $\begin{array}{l}1,732 \pm \\
0,139\end{array}$ \\
\hline A 174 & $\begin{array}{l}9,51 \pm \\
0,24 \\
\end{array}$ & $\begin{array}{l}71,461 \pm \\
2,413 \\
\end{array}$ & $\begin{array}{l}2,183 \pm \\
0,069 \\
\end{array}$ & $\begin{array}{l}0,295 \pm \\
0,086 \\
\end{array}$ & $\begin{array}{l}0,592 \pm \\
0,029 \\
\end{array}$ & $\begin{array}{l}7,138 \pm \\
0,124 \\
\end{array}$ \\
\hline A182 & $\begin{array}{l}8,97 \pm \\
0,12\end{array}$ & $\begin{array}{l}55,268 \pm \\
1,112\end{array}$ & $\begin{array}{l}2,300 \pm \\
0,024\end{array}$ & $\begin{array}{l}0,241 \pm \\
0,045\end{array}$ & $\begin{array}{l}0,545 \pm \\
0,018\end{array}$ & $\begin{array}{l}6,814 \pm \\
0,286\end{array}$ \\
\hline A221 & $\begin{array}{l}8,66 \pm \\
0,108 \\
\end{array}$ & $\begin{array}{l}55,951 \pm \\
1,756 \\
\end{array}$ & $\begin{array}{l}2,678 \pm \\
0,227 \\
\end{array}$ & $\begin{array}{l}0,305 \pm \\
0,029\end{array}$ & $\begin{array}{l}0,618 \pm \\
0,023\end{array}$ & $\begin{array}{l}7,505 \pm \\
0,188 \\
\end{array}$ \\
\hline A222 & $\begin{array}{l}8,26 \pm \\
0,17 \\
\end{array}$ & $\begin{array}{l}65,898 \pm \\
0,515\end{array}$ & $\begin{array}{l}2,591 \pm \\
0,093\end{array}$ & $\begin{array}{l}0,378 \pm \\
0,026\end{array}$ & $\begin{array}{l}0,377 \pm \\
0,126\end{array}$ & $\begin{array}{l}3,181 \pm \\
0,280\end{array}$ \\
\hline A231 & $\begin{array}{l}8,47 \pm \\
0,08 \\
\end{array}$ & $\begin{array}{l}85,755 \pm \\
1,377 \\
\end{array}$ & $\begin{array}{l}3,437 \pm \\
0,267 \\
\end{array}$ & $\begin{array}{l}0,330 \pm \\
0,028\end{array}$ & $\begin{array}{l}0,597 \pm \\
0,028 \\
\end{array}$ & $\begin{array}{l}5,428 \pm \\
0,059 \\
\end{array}$ \\
\hline A 232 & $\begin{array}{l}8,83 \pm \\
0,11 \\
\end{array}$ & $\begin{array}{l}88,278 \pm \\
2,401\end{array}$ & $\begin{array}{l}3,013 \pm \\
0,137\end{array}$ & $\begin{array}{l}0,354 \pm \\
0,049\end{array}$ & $\begin{array}{l}0,582 \pm \\
0,023\end{array}$ & $\begin{array}{l}6,871 \pm \\
0,023\end{array}$ \\
\hline A 243 & $\begin{array}{l}8,68 \pm \\
0,28\end{array}$ & $\begin{array}{l}83,671 \pm \\
3,553\end{array}$ & $\begin{array}{l}2,657 \pm \\
0,169 \\
\end{array}$ & $\begin{array}{l}0,368 \pm \\
0,070\end{array}$ & $\begin{array}{l}0,585 \pm \\
0,002\end{array}$ & $\begin{array}{l}8,364 \pm \\
0,134 \\
\end{array}$ \\
\hline A262 & $\begin{array}{l}8,73 \pm \\
0,19 \\
\end{array}$ & $\begin{array}{l}59,607 \pm \\
0,719 \\
\end{array}$ & $\begin{array}{l}2,649 \pm \\
0,062 \\
\end{array}$ & $\begin{array}{l}0,229 \pm \\
0,052 \\
\end{array}$ & $\begin{array}{l}0,308 \pm \\
0,028 \\
\end{array}$ & $\begin{array}{l}2,997 \pm \\
0,028 \\
\end{array}$ \\
\hline A263 & $\begin{array}{l}8,29 \pm \\
0,35 \\
\end{array}$ & $\begin{array}{l}54,773 \pm \\
1,328\end{array}$ & $\begin{array}{l}2,910 \pm \\
0,219\end{array}$ & $\begin{array}{l}0,275 \pm \\
0,000\end{array}$ & $\begin{array}{l}0,381 \pm \\
0,037\end{array}$ & $\begin{array}{l}3,604 \pm \\
0,116 \\
\end{array}$ \\
\hline A264 & $\begin{array}{l}8,65 \pm \\
0,33 \\
\end{array}$ & $\begin{array}{l}54,860 \pm \\
0,130\end{array}$ & $\begin{array}{l}2,332 \pm \\
0,100\end{array}$ & $\begin{array}{l}0,244 \pm \\
0,055 \\
\end{array}$ & $\begin{array}{l}0,388 \pm \\
0,002 \\
\end{array}$ & $\begin{array}{l}4,201 \pm \\
0,015 \\
\end{array}$ \\
\hline A282 & $\begin{array}{l}8,72 \pm \\
0,08 \\
\end{array}$ & $\begin{array}{l}56,569 \pm \\
2,202\end{array}$ & $\begin{array}{l}2,577 \pm \\
0,164\end{array}$ & $\begin{array}{l}0,199 \pm \\
0,119\end{array}$ & $\begin{array}{l}0,293 \pm \\
0,023\end{array}$ & $\begin{array}{l}2,340 \pm \\
0,162\end{array}$ \\
\hline A291 & $\begin{array}{l}8,24 \pm \\
0,16 \\
\end{array}$ & $\begin{array}{l}39,496 \pm \\
28,054 \\
\end{array}$ & $\begin{array}{l}2,320 \pm \\
0,284 \\
\end{array}$ & $\begin{array}{l}0,308 \pm \\
0,081 \\
\end{array}$ & $\begin{array}{l}0,445 \pm \\
0,048 \\
\end{array}$ & $\begin{array}{l}5,224 \pm \\
0,324 \\
\end{array}$ \\
\hline A313 & $\begin{array}{l}8,38 \pm \\
0,17 \\
\end{array}$ & $\begin{array}{l}70,474 \pm \\
1,472 \\
\end{array}$ & $\begin{array}{l}2,728 \pm \\
0,249 \\
\end{array}$ & $\begin{array}{l}0,296 \pm \\
0,047\end{array}$ & $\begin{array}{l}0,361 \pm \\
0,028\end{array}$ & $\begin{array}{l}2,513 \pm \\
0,067\end{array}$ \\
\hline A 322 & $\begin{array}{l}8,42 \pm \\
0,18 \\
\end{array}$ & $\begin{array}{l}61,990 \pm \\
2,312 \\
\end{array}$ & $\begin{array}{l}2,187 \pm \\
0,157 \\
\end{array}$ & $\begin{array}{l}0,298 \pm \\
0,065 \\
\end{array}$ & $\begin{array}{l}0,339 \pm \\
0,000\end{array}$ & $\begin{array}{l}2,718 \pm \\
0,073 \\
\end{array}$ \\
\hline A331 & $\begin{array}{l}8,41 \pm \\
0,21\end{array}$ & $\begin{array}{l}52,758 \pm \\
2,488\end{array}$ & $\begin{array}{l}2,488 \pm \\
0,068\end{array}$ & $\begin{array}{l}0,353 \pm \\
0,098\end{array}$ & $\begin{array}{l}0,301 \pm \\
0,025\end{array}$ & $\begin{array}{l}1,239 \pm \\
0,042\end{array}$ \\
\hline
\end{tabular}

**: Indican significancia a $\mathrm{p}<0,01$ valores promedio de 3 repeticiones \pm desviación estándar. *** Los valores de azúcares reductores se expresan como equivalentes de glucosa. Los valores de compuestos fenólicos como equivalentes de ácido gálico, GAE. Los valores de flavonoides totales se expresan como equivalentes $\mathrm{mg}$ de catequina. 


\section{Humedad}

Los valores de humedad de las muestras de quinua se encontraron entre 8,24 y $9,51 \%$, el análisis de varianza muestra diferencias altamente significativas con $p<0,01$ para las 24 muestras. Stikic ${ }^{12}$ en la investigación sobre aspectos agronómicos y nutricionales de semillas de quinua reportó valores similares para las muestras utilizadas en la preparación de formulaciones de pan. Por su parte, Repo-Carrasco ${ }^{13}$ en la investigación de granos andinos reportaron valores entre 8,26 y $11,51 \%$ para las semillas de quinua, similares a los encontrados en la investigación para las accesiones.

\section{Azúcares reductores}

Los azúcares son los responsables del dulzor en los alimentos y para la quinua su presencia afecta sensorialmente su aceptación entre los consumidores, así como asegura en las semillas un suministro importante de energía para los procesos de germinación ${ }^{14}$. En la investigación, los resultados en las 24 accesiones revelaron diferencias altamente significativas con $p<0.01$ encontrándose sus valores entre los 30,973 y $88,278 \mathrm{mg} / \mathrm{g}$ de muestra, sobresaliendo la accesión A232. Miranda ${ }^{15}$, en la investigación sobre el impacto del secado en las propiedades nutricionales, compuestos fenólicos y actividad antioxidante de las semillas de quinua de Chile, reveló valores cercanos a $15 \mathrm{mg} / \mathrm{g}$ para sacarosa y alrededor de $12 \mathrm{mg} / \mathrm{g}$ para la glucosa y valores cercanos a $5 \mathrm{mg} / \mathrm{g}$ para fructosa en los experimentos de secado entre 20 y $60{ }^{\circ} \mathrm{C}$, siendo en todos los casos inferiores a los encontrados en la investigación. Por su parte, Ranhotra ${ }^{16}$, en la investigación sobre la composición nutricional, proteínas, minerales, almidón y otros nutrientes en granos perlados del cultivar D407 del Valle de Colorado en Estados Unidos, reportó valores de $27 \mathrm{mg} / \mathrm{g}$ para los azúcares, siendo este ligeramente inferior a los encontrados en la investigación, que identifica semillas con dulzores en equivalentes de glucosa que sobrepasan los $80 \mathrm{mg} / \mathrm{g}$, y cuyas diferencias se deben a las condiciones medioambientales de las zonas de producción, que para el caso de las accesiones corresponden al Altiplano peruano.

\section{Compuestos fenólicos}

Los compuestos fenólicos representan un numeroso grupo de moléculas ampliamente distribuidas en la naturaleza, son componentes importantes de la dieta, y numerosas investigaciones, hoy en día, describen su importancia para la prevención de varias enfermedades y desórdenes fisiológicos, derivados de su estructura química. Su evaluación dentro de las accesiones de quinua se encontraron entre 0,783 y 3,437 mg GAE/g de muestra, sobresaliendo la accesión A231, el análisis de varianza y prueba de significancia de Tukey, muestra diferencias altamente significativas con $p<0.01$ para las diferentes accesiones conservadas bajo condiciones del Altiplano peruano. Abderrahim ${ }^{2}$, en la investigación sobre compuestos fenólicos, betalaínas y capacidad antioxidante para quinuas de color del Altiplano peruano, reportó contenidos de fenólicos libres entre 1,23 y 3,41 mg GAE/g de muestra, inferiores a los encontrados en la investigación y contenidos de 1,89 y 4,52 mg GAE/g de muestra para los fenólicos enlazados, cuyos valores para varias muestras fueron superiores a los hallados en la investigación; diferencia que se explica por la metodología de extracción que contempló medio ácido para los fenólicos libres y enlazados y los tiempos utilizados para su extracción. Por su parte, Dini6 reportó, para las quinuas dulces del Ecuador y amargas del 
Perú, valores de 8,64 mg GAE/g en quinuas amargas y 7,72 mg GAE/g en quinuas dulces, superiores a los presentados en la investigación. Miranda ${ }^{17}$, en la investigación de seis ecotipos de quinua en tres zonas de Chile, reportó valores entre 0,142 y $0,655 \mathrm{mg} \mathrm{GAE} / \mathrm{g}$; mientras que Tang ${ }^{3}$, en la investigación de ecotipos de quinua de color blanco, rojo y negro en Ontario, Canadá, presentó contenidos entre 2 y $3 \mathrm{mg}$ GAE/g para el ecotipo blanco en las fracciones FP (fenólicos libres) y BHP (fenólicos hidrolizables en medio básico); sin embargo, para los ecotipos rojo y negro, los contenidos de fenólicos fueron superiores a los encontrados en la investigación para las accesiones evaluadas.

\section{Flavonoides}

Los flavonoides se describen como compuestos de naturaleza fenólica de bajo peso molecular, que incluyen dentro de su estructura el esqueleto $\mathrm{C}_{6}-\mathrm{C}_{3}-\mathrm{C}_{6}$, dos anillos aromáticos unidos por un anillo heterocíclico pirano, presentan diferentes actividades biológicas debido a la diversidad de patrones de sustitución y variaciones de sus anillos, se clasifican como: 2-fenilbezopiranos, isoflavoides, neoflavonoides, y flavonoides menores como las chalconas $\mathrm{y}$ auronas ${ }^{18}$. La evaluación de estos componentes activos, dentro las accesiones de quinua, revela contenidos que van desde los 0,119 equivalentes $\mathrm{mg}$ de catequina/g hasta los 1,029 equivalentes $\mathrm{mg}$ de catequina/g, sobresaliendo la accesión A124. El análisis de varianza y prueba de significancia de Tukey muestran diferencias altamente significativas con $p<0.01$ para las diferentes accesiones conservadas bajo condiciones del Altiplano peruano, indicando diferentes capacidades a nivel genético para expresar estos componentes activos. Tang ${ }^{3}$, en la investigación de los ecotipos blanco, rojo y negro de quinuas del Canadá, reportó valores cercanos a los 0,5 equivalentes miligramo de catequina/g para las fracciones de FP y BHP, indicando los investigadores que estos componentes activos constituyen una pequeña cantidad de los compuestos fenólicos presentes en las semillas, y cuyos contenidos se encuentran dentro de los presentados en la investigación para las 24 accesiones de color blanco, en cuanto a los ecotipos rojo y negro, estos presentaron contenidos superiores a los presentados en la investigación para las fracciones FP y BHP (cercanos a 1,5 equivalentes mg de catequina/g muestra). Por su parte, Repo-Carrasco ${ }^{13}$, en la investigación de flavonoides y otros compuestos fenólicos en variedades de granos andinos peruanos de quinua, cañihua y kiwicha, reportó valores entre 0,362 y $1,443 \mathrm{mg} / \mathrm{g}$, para los materiales estudiados y donde los flavonoides quercetina, kaempferol, miricetina e isorhamnetina estuvieron presentes.

\section{Betalaínas}

Las betalaínas son compuestos nitrogenados solubles en medio acuosos de acuerdo a su estructura química, estos pigmentos pueden subdividirse como betacianinas de color rojovioleta con máximos de absorción visible en $540 \mathrm{~nm}$, o como betaxantinas de color amarillo con máximos de absorción visible en $480 \mathrm{~nm}$.

Químicamente, las betalaínas son alcaloides derivados de la tirosina que pueden ser de dos tipos: las betacianinas (b) que son de color rojo-violáceo y las betaxantinas (c) anaranjadas amarillentas, ambas con el núcleo fundamental del ácido betalámico (a).

El ácido betalámico (a) es el cromóforo común a todos los pigmentos betalaínicos; las betacianinas tienen un residuo ciclo-DOPA mientras que las betaxantinas tienen aminoácidos 
o aminas adicionadas en dicha posición. Las betacianinas son glicósidos mayormente de la betanidina. Tal como se muestra en la figura 1.

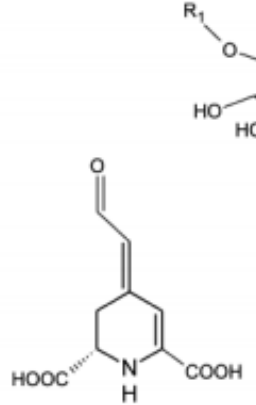

(a)

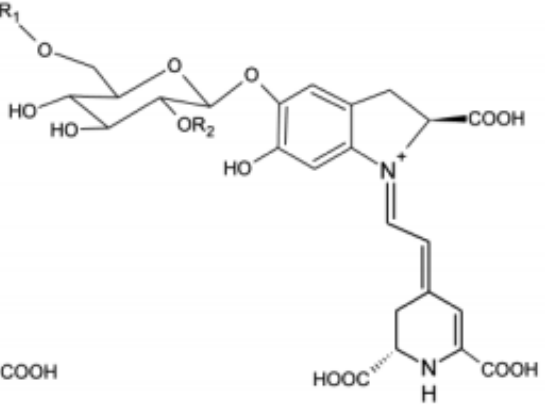

(b)

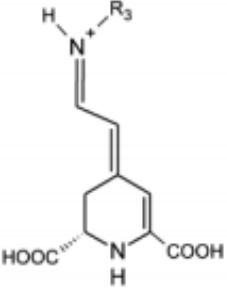

(c)

Figura 1. Estructuras químicas del ácido betalámico (a), betacianinas (b) y betaxantinas (c)

Su evaluación en las accesiones de quinua reveló valores de betacianinas entre 0,278 y 0,883 $\mathrm{mg} / 100 \mathrm{~g}$, y para las betaxantinas entre 1,139 y 13,760 mg/100 g, sobresaliendo en ambos casos la accesión A124. El análisis de varianza y prueba de significancia de Tukey para los resultados de betalaínas en las diferentes muestras reveló diferencias altamente significativas con $p<0.01$ para las diferentes accesiones, lo que significa una alta diversidad de estos componentes activos dentro del material conservado bajo condiciones del Altiplano peruano. Abderrahim $^{2}$, en el estudio de 13 ecotipos rojos y rosados de quinua del Altiplano peruano, encontró valores superiores de betacianinas $(0,15-5,23 \mathrm{mg} / 100 \mathrm{~g})$ a los presentados en la investigación, mientras que los valores de betaxantinas fueron inferiores a los encontrados en la investigación $(0,00-1,63 \mathrm{mg} / 100 \mathrm{~g})$, confirmando los valores experimentales de la investigación para los ecotipos de color blanco.

\section{Capacidad antioxidante}

Actualmente diferentes métodos se han introducido para medir la capacidad antioxidante de alimentos y muestras de origen natural. El concepto que originalmente se definió en química posteriormente se adoptó por la biología, la medicina, la epidemiología, la nutrición, entre otras ciencias; y este describe la habilidad de moléculas de origen natural o sintético para intervenir en procesos redox que estabilicen radicales libres, en ese sentido, beneficios saludables se observan por su consumo ${ }^{19}$.

En alimentos, ensayos del (Acido2,2'-azino-bis-(3-etilbenzotiazolina)-6-sulfonico) (ABTS), (1,1-difeneil-2-picril hidrazilo) (DPPH), Capacidad de absorción de radicales de oxígeno (ORAC), o de la Capacidad férrica de reducción del plasma (FRAP), se utilizan extensivamente y la habilidad de los antioxidantes se monitorean espectrofotométricamente cuando se expresa la capacidad antioxidante en equivalentes Trolox (TEAC), equivalentes de vitamina $\mathrm{C}$ (VCEAC), o como equivalentes de ácido gálico $(\mathrm{GAE})^{20}$. En la investigación, la 
capacidad antioxidante de las 24 accesiones de quinua según DPPH y ABTS, utilizando los extractos $\mathrm{EtOH}-\mathrm{H}_{2} \mathrm{O}(1: 1)$ y $\mathrm{MeOH}-\mathrm{H}_{2} \mathrm{O}(2: 1)$, expresados según equivalentes de ácido gálico (GAE) o equivalentes Trolox (TEAC), la tabla 2 muestra diferencias altamente significativas con $p<0.01$ entre las diferentes muestras.

Tabla 2. Actividad antioxidante de las 24 accesiones de quinua según DPPH y ABTS

\begin{tabular}{|c|c|c|c|c|c|}
\hline \multirow[b]{2}{*}{$\begin{array}{l}\text { Código } \\
\text { Accesión }\end{array}$} & \multicolumn{3}{|c|}{ Actividad Antioxidante según DPPH } & \multicolumn{2}{|c|}{ Actividad Antioxidante según ABTS } \\
\hline & $\begin{array}{l}\text { (mg } \\
\text { equivalente de } \\
\text { ácido gálico } \\
\mathrm{GAE} / \\
100 \mathrm{~g}) \\
* *\end{array}$ & $\begin{array}{l}\mathrm{MeOH}-\mathrm{H}_{2} \mathrm{O} \\
(2: 1)(\mathrm{Trolox} \\
\mu \mathrm{mol} / 100 \mathrm{~g}) \\
* *\end{array}$ & 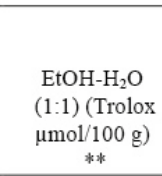 & $\begin{array}{c}\mathrm{MeOH}-\mathrm{H}_{2} \mathrm{O} \\
(2: 1)(\mathrm{Trolox} \\
\mu \begin{array}{c}\mu \mathrm{mol} / 100 \mathrm{~g}) \\
* *\end{array}\end{array}$ & $\begin{array}{c}\text { EtOH- } \mathrm{H}_{2} \mathrm{O} \\
(1: 1)(\mathrm{Trolox} \\
\mu \mathrm{mol} / 100 \mathrm{~g}) \\
* *\end{array}$ \\
\hline B381 & $\begin{array}{l}66,312 \pm \\
0,021\end{array}$ & $\begin{array}{l}511,888 \pm \\
39,588\end{array}$ & $\begin{array}{l}637,369 \pm \\
18,265 \\
\end{array}$ & $\begin{array}{l}1083,420 \pm \\
58,148\end{array}$ & $\begin{array}{l}916,496 \pm \\
7,988\end{array}$ \\
\hline A114 & $\begin{array}{l}55,683 \pm \\
0,022\end{array}$ & $\begin{array}{l}550,616 \pm \\
3,277\end{array}$ & $\begin{array}{l}709,056 \pm \\
4,280\end{array}$ & $\begin{array}{l}1456,852 \pm \\
36,777 \\
\end{array}$ & $\begin{array}{l}1312,244 \pm \\
8,601\end{array}$ \\
\hline A121 & $\begin{array}{l}67,739 \pm \\
0,018\end{array}$ & $\begin{array}{l}668,945 \pm \\
19,797\end{array}$ & $\begin{array}{l}807,484 \pm \\
51,878\end{array}$ & $\begin{array}{l}1374,606 \pm \\
28,258\end{array}$ & $\begin{array}{l}1217,648 \pm \\
7,271\end{array}$ \\
\hline A122 & $\begin{array}{l}55,402 \pm \\
0,020\end{array}$ & $\begin{array}{l}533,261 \pm \\
48,465\end{array}$ & $\begin{array}{l}760,554 \pm \\
21,462\end{array}$ & $\begin{array}{l}1067,399 \pm \\
143,945\end{array}$ & $\begin{array}{l}978,679 \pm \\
16,763\end{array}$ \\
\hline A123 & $\begin{array}{l}15,960 \pm \\
0,013\end{array}$ & $\begin{array}{l}972,875 \pm \\
53,896\end{array}$ & $\begin{array}{l}1195,746 \pm \\
18,086\end{array}$ & $\begin{array}{l}1699,126 \pm \\
48,175 \\
\end{array}$ & $\begin{array}{l}1481,363 \pm \\
13,146\end{array}$ \\
\hline A124 & $\begin{array}{l}81,851 \pm \\
0,001\end{array}$ & $\begin{array}{l}611,383 \pm \\
19,803\end{array}$ & $\begin{array}{l}711,659 \pm \\
17,619\end{array}$ & $\begin{array}{l}1835,784 \pm \\
69,060\end{array}$ & $\begin{array}{l}1507,938 \pm \\
30,316\end{array}$ \\
\hline A134 & $\begin{array}{l}48,037 \pm \\
0,047\end{array}$ & $\begin{array}{l}621,963 \pm \\
39,678\end{array}$ & $\begin{array}{l}767,740 \pm \\
13,681\end{array}$ & $\begin{array}{l}1550,885 \pm \\
39,614\end{array}$ & $\begin{array}{l}1229,184 \pm \\
17,745\end{array}$ \\
\hline A 142 & $\begin{array}{l}53,366 \pm \\
0,023\end{array}$ & $\begin{array}{l}584,205 \pm \\
96,839\end{array}$ & $\begin{array}{l}683,597 \pm \\
13,654\end{array}$ & $\begin{array}{l}1535,630 \pm \\
206,336\end{array}$ & $\begin{array}{l}1261,218 \pm \\
27,701\end{array}$ \\
\hline A163 & $\begin{array}{l}58,813 \pm \\
0,015\end{array}$ & $\begin{array}{l}482,883 \pm \\
55,048\end{array}$ & $\begin{array}{l}621,956 \pm \\
9,908\end{array}$ & $\begin{array}{l}1154,960 \pm \\
92,205\end{array}$ & $\begin{array}{l}884,050 \pm \\
8,400\end{array}$ \\
\hline A174 & $\begin{array}{l}40,772 \pm \\
0,071\end{array}$ & $\begin{array}{l}566,291 \pm \\
119,200\end{array}$ & $\begin{array}{l}679,626 \pm \\
7,430\end{array}$ & $\begin{array}{l}1440,688 \pm \\
288,625\end{array}$ & $\begin{array}{l}1193,602 \pm \\
9,088\end{array}$ \\
\hline A182 & $\begin{array}{l}46,774 \pm \\
0,015\end{array}$ & $\begin{array}{l}520,557 \pm \\
47,117\end{array}$ & $\begin{array}{l}623,491 \pm \\
11,598\end{array}$ & $\begin{array}{l}1477,112 \pm \\
30,229\end{array}$ & $\begin{array}{l}1123,443 \pm \\
12,214\end{array}$ \\
\hline A221 & $\begin{array}{l}53,671 \pm \\
0,012\end{array}$ & $\begin{array}{l}570,711 \pm \\
10,680\end{array}$ & $\begin{array}{l}709,780 \pm \\
10,197\end{array}$ & $\begin{array}{l}1660,205 \pm \\
90,000\end{array}$ & $\begin{array}{l}1243,487 \pm \\
6,834\end{array}$ \\
\hline A222 & $\begin{array}{l}55,850 \pm \\
0,095\end{array}$ & $\begin{array}{l}532,143 \pm \\
37,748\end{array}$ & $\begin{array}{l}729,441 \pm \\
14,148\end{array}$ & $\begin{array}{l}1036,549 \pm \\
137,924\end{array}$ & $\begin{array}{l}986,307 \pm \\
7,255\end{array}$ \\
\hline A231 & $\begin{array}{l}49,310 \pm \\
0,024\end{array}$ & $\begin{array}{l}474,215 \pm \\
17,545\end{array}$ & $\begin{array}{l}657,858 \pm \\
6,161\end{array}$ & $\begin{array}{l}1312,406 \pm \\
21,746\end{array}$ & $\begin{array}{l}1212,242 \pm \\
6,562\end{array}$ \\
\hline A232 & $\begin{array}{l}52,272 \pm \\
0,041\end{array}$ & $\begin{array}{l}475,493 \pm \\
28,102\end{array}$ & $\begin{array}{l}696,585 \pm \\
7,719\end{array}$ & $\begin{array}{l}1245,006 \pm \\
215,591\end{array}$ & $\begin{array}{l}1194,501 \pm \\
14,985\end{array}$ \\
\hline A243 & $\begin{array}{l}46,563 \pm \\
0,025\end{array}$ & $\begin{array}{l}483,368 \pm \\
9,813\end{array}$ & $\begin{array}{l}643,041 \pm \\
11,249\end{array}$ & $\begin{array}{l}1224,858 \pm \\
65,419\end{array}$ & $\begin{array}{l}1077,280 \pm \\
8,450\end{array}$ \\
\hline A262 & $\begin{array}{l}56,409 \pm \\
0,006\end{array}$ & $\begin{array}{l}558,233 \pm \\
27,308\end{array}$ & $\begin{array}{l}697,989 \pm \\
3,363\end{array}$ & $\begin{array}{l}1122,087 \pm \\
101,242\end{array}$ & $\begin{array}{l}895,788 \pm \\
16,729\end{array}$ \\
\hline A263 & $\begin{array}{l}54,935 \pm \\
0,031\end{array}$ & $\begin{array}{l}538,184 \pm \\
22,224\end{array}$ & $\begin{array}{l}735,060 \pm \\
15,316\end{array}$ & $\begin{array}{l}1083,805 \pm \\
18,133\end{array}$ & $\begin{array}{l}928,507 \pm \\
17,371\end{array}$ \\
\hline A264 & $\begin{array}{l}70,233 \pm \\
0,020\end{array}$ & $\begin{array}{l}683,746 \pm \\
14,450 \\
\end{array}$ & $\begin{array}{l}791,403 \pm \\
10,770\end{array}$ & $\begin{array}{l}1368,399 \pm \\
48,262\end{array}$ & $\begin{array}{l}1065,087 \pm \\
6,395\end{array}$ \\
\hline A282 & $\begin{array}{l}49,670 \pm \\
0,055\end{array}$ & $\begin{array}{l}570,269 \pm \\
16,073\end{array}$ & $\begin{array}{l}486,080 \pm \\
3,572\end{array}$ & $\begin{array}{l}1212,965 \pm \\
30,197\end{array}$ & $\begin{array}{l}878,444 \pm \\
23,482 \\
\end{array}$ \\
\hline A291 & $\begin{array}{l}56,054 \pm \\
0,013\end{array}$ & $\begin{array}{l}528,280 \pm \\
37,219\end{array}$ & $\begin{array}{l}525,398 \pm \\
8,364\end{array}$ & $\begin{array}{l}1330,621 \pm \\
57,013\end{array}$ & $\begin{array}{l}1056,143 \pm \\
34,274\end{array}$ \\
\hline A 313 & $\begin{array}{l}44,953 \pm \\
0,009\end{array}$ & $\begin{array}{l}550,316 \pm \\
19,198\end{array}$ & $\begin{array}{l}521,644 \pm \\
5,462\end{array}$ & $\begin{array}{l}1412,472 \pm \\
54,852\end{array}$ & $\begin{array}{l}1017,935 \pm \\
12,551\end{array}$ \\
\hline A 322 & $\begin{array}{l}54,072 \pm \\
0,019\end{array}$ & $\begin{array}{l}611,525 \pm \\
97,303\end{array}$ & $\begin{array}{l}586,178 \pm \\
4,012\end{array}$ & $\begin{array}{l}1376,976 \pm \\
132,593\end{array}$ & $\begin{array}{l}1071,643 \pm \\
14,699\end{array}$ \\
\hline A331 & $\begin{array}{l}52,195 \pm \\
0,030\end{array}$ & $\begin{array}{l}579,909 \pm \\
30,177\end{array}$ & $\begin{array}{l}602,531 \pm \\
8,448\end{array}$ & $\begin{array}{l}1373,555 \pm \\
41,764\end{array}$ & $\begin{array}{l}966,700 \pm \\
11,671\end{array}$ \\
\hline
\end{tabular}

**: Indican significancia a $\mathrm{p}<0,01$, valores promedio de 4 repeticiones \pm desviación estándar. 
En el caso de capacidad antioxidante según DPPH en GAE de los extractos EtOH- $\mathrm{H}_{2} \mathrm{O}(1: 1)$ se observaron valores entre 40,772 y 81,851 mg GAE/100 g; mientras que como TEAC los valores estuvieron entre los 486,080 y 1195,746 equivalentes de Trolox $\mu \mathrm{mol} / 100 \mathrm{~g}$, sobresaliendo la accesión A123, y cuya capacidad coincidió con la capacidad antioxidante del extracto $\mathrm{MeOH}-\mathrm{H}_{2} \mathrm{O}$ (2:1) según DPPH y expresado como TEAC, Fischer ${ }^{8}$ en la investigación sobre la capacidad antioxidante de variedades chilenas de quinua sometidas a diferentes condiciones de estrés hídrico encontraron valores entre 320 y $510 \mathrm{mg}$ GAE/100 g para el genotipo AG 2010, superiores a los genotipos Regalona y B080; estos resultados muestran un efecto positivo del estrés hídrico sobre la expresión de metabolitos responsables de la capacidad antioxidante, los que fueron significativamente superiores a los encontrados en la investigación. Por su parte $\mathrm{Tang}^{3}$, en la investigación de quinuas blanca, roja y negra en diferentes fracciones de extracción de fenólicos libres, (FP), fenólicos libres en medio básico (BHP) y fenólicos hidrolizables de naturaleza ácida (AHP) según DPPH en TEAC, reportó valores entre los 600 y $1100 \mu \mathrm{mol}$ Trolox/100 g, para los FP, BHP y AHP en los ecotipos estudiados y los que concuerdan con los valores hallados en la investigación para las 24 accesiones de quinua blanca; sin embargo, para los ecotipos de colores, como el negro, los valores alcanzaron los $1400 \mu \mathrm{mol}$ Trolox/100 g en la fracción AHP, donde las condiciones de extracción fueron en medio ácido. Por su parte, Hirose $^{21}$, en la investigación de las propiedades antioxidantes de las semillas de quinua cultivadas en Japón, reporta valores entre 502 y 950 $\mu \mathrm{mol}$ Trolox $/ 100 \mathrm{~g}$, que se encontraron dentro de los valores presentados en la investigación para los extractos $\mathrm{MeOH}-\mathrm{H}_{2} \mathrm{O}(2: 1)$ y EtOH- $\mathrm{H}_{2} \mathrm{O}(1: 1)$. Dini ${ }^{6}$, al evaluar el efecto de la cocción sobre los ecotipos dulces y amargos de semillas de quinua, determinó el efecto negativo de la cocción sobre las semillas, los valores encontrados por los investigadores estuvieron según DPPH entre 287 y $670 \mu \mathrm{mol}$ Trolox/100 g, los que fueron inferiores a los presentados en las 24 accesiones del INIA. Para el caso de la actividad antioxidante, según ABTS, se prepararon extractos $\mathrm{MeOH}-\mathrm{H}_{2} \mathrm{O}(2: 1)$ y EtOH- $\mathrm{H}_{2} \mathrm{O}(1: 1)$ y evaluó la capacidad antioxidante en TEAC, tabla 2, los resultados muestran valores entre los 1036,549 y $1835,784 \mu \mathrm{mol}$ Trolox $/ 100 \mathrm{~g}$ para $\mathrm{MeOH}-\mathrm{H}_{2} \mathrm{O}(2: 1)$, y 878,444 y $1507,938 \mu \mathrm{mol}$ Trolox/100 g para el extracto EtOH- $\mathrm{H}_{2} \mathrm{O}$ (1:1), sobresaliendo en ambos extractos la accesión A124.

Con respecto a las relaciones que existen entre las evaluaciones de capacidad antioxidante, según DPPH y ABTS, de los diferentes extractos de las accesiones y la forma como estos se expresan en GAE o TEAC, así como con los fenólicos y flavonoides, en la tabla 3 se presentan análisis de correlación según Pearson.

Tabla 3. Coeficientes de correlación de Pearson entre las mediciones de capacidad antioxidante según DPPH y ABTS, compuestos fenólicos y flavonoides.

\begin{tabular}{|c|c|c|c|c|c|c|c|}
\hline & $\begin{array}{c}\text { DPPH } \\
(1: 1 \mathrm{GAE})\end{array}$ & $\begin{array}{c}\text { DPPH } \\
(1: 1 \text { TEAC) }\end{array}$ & $\begin{array}{c}\text { ABTS } \\
(1: 1 \text { TEAC) }\end{array}$ & $\begin{array}{c}\text { DPPH } \\
(2: 1 \text { TEAC) }\end{array}$ & $\begin{array}{c}\text { ABTS } \\
(2: 1 \text { TEAC) }\end{array}$ & Fenólicos & Flavonoides \\
\hline DPPH (1:1 GAE) & 1,00 & $-0,35$ & $-0,14$ & $-0,39$ & $-0,12$ & 0,54 & 0,20 \\
\hline DPPH (1:1 TEAC) & $-0,35$ & 1,00 & 0,55 & 0,79 & 0,31 & $-0,46$ & 0,35 \\
\hline ABTS (1:1 TEAC) & $-0,14$ & 0,55 & 1,00 & 0,51 & 0,85 & 0,02 & 0,57 \\
\hline DPPH (2:1 TEAC) & $-0,39$ & 0,79 & 0,51 & 1,00 & 0,53 & $-0,59$ & 0,28 \\
\hline $\operatorname{ABTS}(2: 1$ TEAC) & $-0,12$ & 0,31 & 0,85 & 0,53 & 1,00 & $-0,06$ & 0,48 \\
\hline Fenólicos & 0,54 & $-0,46$ & 0,02 & $-0,59$ & $-0,06$ & 1,00 & 0,06 \\
\hline Flavonoides & 0,20 & 0,35 & 0,57 & 0,28 & 0,48 & 0,06 & 1,00 \\
\hline
\end{tabular}


DPPH (1:1 GAE): representa actividad antioxidante de extractos EtOH- $\mathrm{H}_{2} \mathrm{O}$ (1:1) según DPPH expresado como equivalentes de ácido gálico (GAE). DPPH (1:1 TEAC): representa la actividad antioxidante de extractos EtOH- $\mathrm{H}_{2} \mathrm{O}(1: 1)$ según DPPH en equivalentes Trolox. ABTS (1:1 TEAC): representa la actividad antioxidante de extractos EtOH- $\mathrm{H}_{2} \mathrm{O}(1: 1)$ según ABTS en equivalentes Trolox. DPPH (2:1 TEAC): representa la actividad antioxidante de extractos $\mathrm{MeOH}-\mathrm{H}_{2} \mathrm{O}(2: 1)$ según DPPH en equivalentes Trolox. ABTS (2:1 TEAC): representa la actividad antioxidante de extractos $\mathrm{MeOH}-\mathrm{H}_{2} \mathrm{O}$ (2:1) según ABTS en equivalentes Trolox. Fenólicos: representa compuestos fenólicos expresado como equivalentes mg de ácido gálico (GAE). Flavonoides: representa los flavonoides totales expresado como equivalentes mg de catequina.

El análisis de correlación indica correlaciones muy altamente significativas $p \leq 0,001$ para los valores de capacidad antioxidante según DPPH en TEAC de los extractos EtOH-H $\mathrm{O}_{2}(1: 1)$ y $\mathrm{MeOH}-\mathrm{H}_{2} \mathrm{O}(2: 1)$, así como para los valores de capacidad antioxidante según ABTS en TEAC de los extractos EtOH- $\mathrm{H}_{2} \mathrm{O}(1: 1)$ y $\mathrm{MeOH}-\mathrm{H}_{2} \mathrm{O}(2: 1)$. Correlaciones altamente significativas con $p \leq 0,01$ se observaron para la capacidad antioxidante según DPPH de los extractos EtOH$\mathrm{H}_{2} \mathrm{O}(1: 1)$ y capacidad antioxidante según ABTS de los extractos $\mathrm{MeOH}-\mathrm{H}_{2} \mathrm{O}(2: 1)$, ambos en TEAC, que está de acuerdo con lo que estableció Floege ${ }^{19}$ en la comparación de capacidades antioxidantes, según DPPH y ABTS, en equivalentes de ácido ascórbico de frutos, vegetales y bebidas del mercado de Estados Unidos, donde establecieron correlaciones significativas de acuerdo a Spearman para los valores encontrados en las diferentes fuentes.

En el caso de correlaciones entre capacidad antioxidante y compuestos fenólicos, esta fue altamente significativa con $p \leq 0,01$ para las capacidades antioxidantes de los extractos EtOH$\mathrm{H}_{2} \mathrm{O}$ (1:1) según DPPH en GAE y los compuestos fenólicos, en ese sentido Floegel ${ }^{19}$ llegó a las mismas conclusiones; mientras que para la capacidad antioxidante de los extractos $\mathrm{MeOH}-$ $\mathrm{H}_{2} \mathrm{O}(2: 1)$ en TEAC y los compuestos fenólicos la correlación fue altamente significativa de modo negativo. En el caso de las correlaciones entre capacidad antioxidante y los flavonoides, se observó una correlación altamente significativa $p \leq 0,01$ para la capacidad antioxidante de los extractos EtOH- $\mathrm{H}_{2} \mathrm{O}$ (1:1) según ABTS en TEAC y los flavonoides; así como una correlación significativa $\mathrm{p} \leq 0.05$ de la capacidad antioxidante según ABTS de los extractos $\mathrm{MeOH}-\mathrm{H}_{2} \mathrm{O}$ (2:1) en TEAC y los flavonoides; para este caso, Floegel ${ }^{19}$, de acuerdo a los coeficientes de correlación según Spearman, reporta valores de 0,718 para flavonoides con ABTS y 0,708 para flavonoides y DPPH, recordando que los investigadores expresaron sus resultados como VCEAC. Para las betalaínas y betaxantinas evaluados en la investigación, se observaron correlaciones altamente significativas $p \leq 0,001$ para los valores de capacidad antioxidante de los extractos EtOH- $\mathrm{H}_{2} \mathrm{O}(1: 1)$ y MeOH- $\mathrm{H}_{2} \mathrm{O}(2: 1)$ según ABTS en TEAC con los extractos obtenidos en medio buffer $\mathrm{pH}$ 6,5 para las betalaínas y betaxantinas, información que no se presenta en la tabla 3, que para la investigación son de importancia debido a que no existen antecedentes al respecto en semillas de quinua. Para el análisis de correlación entre las betalaínas y las betaxantinas los resultados de las evaluaciones presentan una correlación altamente significativa $p \leq 0,001$, en relación con los flavonoides se observaron correlaciones significativas (betacianinas, $p \leq 0,001$; betaxantinas, $p \leq 0,01$ ).

Las variaciones obtenidas de las actividades antioxidantes en nuestro estudio podría ser el resultado de las distintas polaridades de los disolventes empleados, ya que condujo a la 
extracción de un grupo seleccionado de compuestos antioxidantes en función de su estructura química, polaridad y solubilidad, afectando la capacidad antioxidante entre los extractos, como lo reportado por Zhi-feng $\mathrm{Fu}^{22}$ en su estudio de actividad antioxidante en hojas de camote (Ipomoea batatas L.) extraídas con solventes de varias polaridades, ellos estudiaron diez solventes diferentes, incluyendo agua, metanol acuoso, etanol acuoso y acetona acuosa, y el efecto de los solventes sobre la recuperación de las actividades antioxidantes en hojas de camote. El rendimiento de las actividades antioxidantes de los extractos se vio afectado significativamente por la polaridad del disolvente de extracción.

\section{CONCLUSIONES}

- Los azúcares reductores en las semillas de quinua se encontraron entre los 30,973 y $88,278 \mathrm{mg}$ glucosa/g, sobresaliendo la accesión A232. En compuestos fenólicos los resultados alcanzaron los 3,437 mg GAE/g, mientras que para los flavonoides los 1,029 equivalentes $\mathrm{mg}$ de catequina/g. A nivel de compuestos nitrogenados, las betacianinas se encontraron entre los 0.278 y $0.883 \mathrm{mg} / 100 \mathrm{~g}$, mientras que las betaxantinas entre 1,139 y 13,760 mg/100 g, sobresaliendo en ambos casos la accesión A124.

- La capacidad antioxidante según DPPH y ABTS de los extractos EtOH- $\mathrm{H}_{2} \mathrm{O}$ (1:1) y $\mathrm{MeOH}-\mathrm{H} 2 \mathrm{O}$ (2:1), expresada como GAE o TEAC en las diferentes accesiones, reporta valores significativos para la accesión A123 en TEAC tanto para DPPH y ABTS, en el caso de GAE sobresalió la A124.

- La correlación de Pearson reveló correlaciones muy altamente significativas $(p \leq 0,001)$ entre los valores de capacidad antioxidante según DPPH y ABTS de los extractos $\left(\right.$ EtOH- $\mathrm{H}_{2} \mathrm{O}(1: 1)$ y $\left.\mathrm{MeOH}-\mathrm{H}_{2} \mathrm{O}(2: 1)\right)$ en TEAC, la capacidad antioxidante según DPPH en GAE se correlacionó de manera significativa con los compuestos fenólicos, y la capacidad antioxidante de los extractos EtOH- $\mathrm{H}_{2} \mathrm{O}(1: 1)$ según ABTS en TEAC se correlacionó significativamente con los flavonoides. Las betalaínas y betaxantinas presentaron correlaciones muy altamente significativas $(p \leq 0,001)$, mientras que estos compuestos nitrogenados se correlacionaron de manera significativa con los flavonoides (betacianinas, $p \leq 0.001$; betaxantinas, $p \leq 0.01$ ).

\section{AGRADECIMIENTO}

Al Instituto Nacional de Innovación Agraria INIA, en especial al Ing. Fredy Quispe por su apoyo y confianza en este proyecto durante estos años de investigación, del mismo modo a la Ing. Karina Ccapa por su apoyo y amistad.

A la Asociación Universitaria Iberoamericana de Postgrado AUIP por la beca concedida para la realización de la Tesis Doctoral en la Universidad de Córdoba de España.

Al Departamento de Bromatología y Tecnología de los Alimentos de la Universidad de Córdoba de España, en especial a los Doctores Fernando Cámara y Rafael Moreno. 


\section{REFERENCIAS BIBLIOGRÁFICAS}

1. Dueñas D. Vigilancia competitiva de la quinua: potencialidad para el departamento de Boyacá. Suma de Negocios. 2014; 12: 85-95.

2. Abderrahim F, Huanatico E, Segura R, Arribas S, Gonzales M, Condezo-Hoyos L. Physical features, phenolic compounds, betalains and total antioxidant capacity of coloured quinoa seeds (Chenopodium quinoa Willd.) from Peruvian Altiplano. Food Chem. 2015; 183: 83-90.

3. Tang Y, Li X, Zhang B, Chen P, Liu R, Tsao T. Characterisation of phenolics, betanins and antioxidant activities in seeds of three Chenopodium quinoa Willd. Genotypes. Food Chem. 2015; 166: 380-388.

4. Normas Mexicanas. NMX-F-428-1982. Alimentos. Determinación de humedad (método rápido de la termobalanza). Normas mexicanas. Dirección general de normas. 1982.

5. Najmus A, Whitney P. Differential behaviour of the dinitrosalicylic acid (DNS) reagent towards mono-and di-saccharide sugars. Biomass Bioenergy. 2011; 35: 4748-4750.

6. Dini I, Tenore G, Dini A. Antioxidant compound contents and antioxidant activity before and after cooking in sweet and bitter Chenopodium quinoa seeds. LWT - Food Sci Technol. 2010; 43: 447-451.

7. Quispe F, Arroyo K, Gorriti G. Características morfológicas y químicas de 3 cultivares de maíz morado (Zea mays L.) en Arequipa - Perú. Rev Soc Quím Perú. 2011; 77 (3):205-217.

8. Fisher S, Wilckens R, Jara J, Aranda M. Variation in antioxidant capacity of quinoa (Chenopodium quinoa Will) subjected to drought stress. Ind Crops Prod. 2013; 46, 341349.

9. Re R, Pellegrini N, Proteggente A, Pannala A, Yang M, Rice-Evans C. Antioxidant activity applying and improved ABTS radical cation decolorization assay. Free Radic Biol Med. 1999; 26(9-10): 1231-7.

10. Von Elbe JH. Betalains. CPFAC. 2001; Unit F3.1.3-F3.1.7. DOI: 10.1002/0471142913. faf0301s00.

11. Di Rienzo JA, Casanoves F, Balzarini MG, Gonzalez L, Tablada M, Robledo CW. InfoStat versión 2015. Grupo InfoStat, FCA, Universidad Nacional de Córdoba, Argentina; 2015.

12. Stikic R, Glamoclija D, Demin M, Vucelic-Radovic B, Jovanovic Z, MilojkovicOpsenica D, Jacobsen S, Milovanovic M. Agronomical and nutritional evaluation of quinoa seeds (Chenopodium quinoa Willd.) as an ingredient in bread formulations. J Cereal Sci. 2012; 55: 132-138.

13. Repo-Carrasco-Valencia R, Hellström JK, Pihlava J, Mattila P. Flavonoids and other phenolic compounds in Andean indigenous grains: Quinoa (Chenopodium quinoa), kañiwa (Chenopodium pallidicaule) and kiwicha (Amaranthus caudatus). Food Chem. $2010 ; 120,128-133$.

14. Weber H, Borisjuk L, Wobus U. Sugar import and metabolism during seed development. Trends Plant Sci. 1997; 2(5): 169-174.

15. Miranda M, Vega-Gálvez A, López J, Parada G, Sanders M, Aranda M, et al. Impact of air-drying temperature on nutritional properties, total phenolic content and antioxidant 
capacity of quinoa seeds (Chenopodium quinoa Willd). Ind Crops Prod. 2010; 32: 258263.

16. Ranhotra GS, Gelroth JA, Glaser BK, Lorenz KJ, Johnson DL. Composition and Protein Nutritional Quality of Quinoa. Cereal Chem. 1993; 70(3): 303-305.

17. Miranda M, Vega-Gálvez A, Uribe E, López J, Martínez E, Rodríguez M, Quispe I, Di Scala, K. Physico-chemical analysis, antioxidant capacity and vitamins of six ecotypes of Chilean quinoa (Chenopodium quinoa Willd). Procedia Food Sci. 2011. 1:14391446.

18. Marais JPJ, Deavours B, Dixon RA, Ferreira D. The stereochemistry of flavonoids In Grotewold E, editor. The Science of Flavonoids. New York: Springer Press; 2007. p. $1-35$.

19. Floegel A, Kim D, Chung S, Koo S, Chun O. Comparison of ABTS/DPPH assays to measure antioxidant capacity in popular antioxidant-rich US foods. J Food Comp Anal. 2011; 24: 1043-1048.

20. Rodriguez-Amaya DB. Quantitative analysis, in vitro assessement of bio-availibility and antioxidant activity of food carotenoids-A review. J Food Comp Anal. 2010; 23: 764-740.

21. Hirose Y, Fujita T, Ishii T, Ueno N. Antioxidative properties and flavonoid composition of Chenopodium quinoa seeds cultivated in Japan. Food Chem. 2010; 119: 1300-1306.

22. Zhi-feng F, Zong-cai T, Lu Z, Hui W, Qing-hui W, Tao H. Antioxidant activities and polyphenols of sweet potato (Ipomoea batatas L.) leaves extracted with solvents of various polarities. Food Biosci. 2016; 15: 11-18 